\title{
Tool Wear Monitoring in Turning Processes Using Vibratory Analysis
}

\author{
Wafaa Rmili \\ Polytech'Tours, Universit Franois Rabelais de Tours, Laboratoire de Mcanique et Rhologie EA 2640, 7 avenue \\ Marcel Dassault, 37200 Tours, France.
}

\author{
Abdeljalil Ouahabi \\ Polytech'Tours, Universit Franois Rabelais de Tours, Signal and Image Group, 7 avenue Marcel Dassault, 37200 \\ Tours, France.
}

\section{Roger Serra}

ENI Val de Loire, Universit Franois Rabelais de Tours, Laboratoire de Mcanique et Rhologie EA 2640, Rue de la chocolaterie, BP 3410, 41034 Blois Cedex, France.

\section{Mecheri Kious \\ Polytech'Tours, Universit Franois Rabelais de Tours, Signal and Image Group, 7 avenue Marcel Dassault, 37200 Tours, France.}

(Received 3 April 2008; accepted 20 January 2009)

\begin{abstract}
The main objective of this paper is to develop a signal processing strategy using vibratory signals in order to provide an efficient tool wear monitoring system able to increase machining performance. The method is based on the changes in the vibration signatures acquired during the turning operation over the tool life. Several signal processing techniques based on time and frequency domain analysis are proposed in order to extract a large number of indicators of the cutting tool state as variance, kurtosis, skewness, and coherence function. In this work, one of the innovative results is the tracking of tool wear by variance and coherence estimation. All of these indicators are correlated and validated by using white light interferometry measurements.
\end{abstract}

This paper focuses on the technologies used in monitoring conventional cutting operations and presents important findings related to this field.

\section{INTRODUCTION}

Machining operations such as turning and milling are used in routine industrial manufacturing processes to realize the machined workpiece under specific characteristics (geometry, surface roughness, etc.). This process is based on removing small pieces by means of a cutting tool. The cutting tool is in contact with the workpiece and the chip along a very small area of a few square millimetres. The temperature and the pressure within the contact area are significantly high (about $1200^{\circ} \mathrm{C}$ and $3000 \mathrm{~N} / \mathrm{mm}^{2}$ ), which makes wear of the cutting tool inevitable. $^{1}$

In these conditions, tool wear is caused by adhesion, abrasion, erosion, diffusion, or other mechanisms. ${ }^{2}$ Cutting tool failure appears in several wear forms: flank wear, crater wear, chipping, etc. These forms depend essentially on cutting tool characteristics, workpiece material, cutting conditions, and types of machining. ${ }^{3}$

This phenomenon decreases the quality of machining because of a loss in surface integrity, an amplification of chatter, and possibly damage to the workpiece. Moreover, dimensional and roughness accuracies of the final product are not optimal. ${ }^{4}$ For these reasons, it is necessary to develop a monitoring system in order to detect and evaluate the tool wear during the cutting process.

Generally, monitoring tool wear can be categorized into direct and indirect methods. The direct methods, which consist of measuring directly the state of the tool using classical optical systems such as CCD-based cameras and ihite light interferometry, evaluate the cutting tool life and measure the crater wear. ${ }^{4,5}$ Recently, K.Niranjan Prasad et al. ${ }^{6}$ proposed to measure the crater wear in three dimensions by means of a stereovision system based on acquiring images during machining. Indirect techniques available in the literature are based on acoustic emission, ${ }^{7,8}$ cutting force measurement ${ }^{9}$ and vibration measurement. ${ }^{10-13}$ In the latter technique, friction between the workpiece, the chip, and the cutting tool generates mechanical vibrations, and they are correlated to the cutting tool state.

The tool wear monitoring techniques, in principle, measure or track the predominant wear generated in specific cutting 